\title{
Concepción en inseminación artificial a tiempo fijo con y sin hormona gonadotrofina coriónica equina.
}

\section{Conception in fixed-time artificial insemination with and without equine chorionic gonadotropin hormone}

\author{
Gudelio Alva Tafur ${ }^{1}$, Nilton Luis Murga Valderrama²
}

\section{RESUMEN}

El objetivo de la presente investigación fue determinar la tasa de concepción en inseminación artificial a tiempo fijo con y sin hormona gonadotrofina coriónica equina (eCG). El experimento contó con 42 vacas de 1 a 4 partos se dividieron en 2 tratamientos: "T1" protocolo con eCG $(\mathrm{n}=21)$ y "T2" protocolo sin eCG $(\mathrm{n}=21)$ (protocolo modificado de Gabriel Bó). Para el “T1" el día de inicio se colocó un dispositivo intravaginal DIB", luego se aplicó $0.8 \mathrm{ml}$ de benzoato de estradiol $\left(\right.$ ESTROVET $^{\circledR)}$ ). El día 8 , se retiró el dispositivo DIB $^{\circledR}$ más $2 \mathrm{ml}$ de prostaglandina $\left(\mathrm{SINCROCIO}^{\circledR}\right)$ más $0.5 \mathrm{ml}$ de cipionato de estradiol (ESTROGAL ${ }^{\circledR}$ ) y $2 \mathrm{ml}(\mathrm{eCG})\left(\mathrm{NOVORMON}^{\circledR}\right)$. El “T2", fue similar al protocolo anterior con la diferencia de que no se suministró hormona (eCG) (NOVORMON $\left.{ }^{\circledR}\right) .50$ horas después de retirado el dispositivo se insemino y se aplicó $2 \mathrm{ml}$ de $\mathrm{GnRh}$ (CONCEPTASE). 40 días después se hizo diagnóstico de preñez con ultrasonografía. Se hizo una evaluación con un cuadro anova. Los resultados sugieren que no existe diferencia estadística significativa entre los grupos tratados, pero si diferencias numéricas a favor del tratamiento 1. En conclusión, el uso de hormona gonadotrofina coriónica equina eleva el porcentaje de preñez.

Palabras clave: Ciclo estral, protocolo, ovulación, celo

\begin{abstract}
The objective of this research was to determine the conception rate in fixed-time artificial insemination with and without equine chorionic gonadotropin hormone (eCG). The experiment consisted of 42 cows from 1 to 4 calving they were divided into 2 treatments: "T1" protocol with eCG $(n=21)$ and "T2" protocol without eCG $(n=21)$. For the "T1" on the start day, a DIB $\AA$ intravaginal device was placed, then $0.8 \mathrm{ml}$ of estradiol benzoate (ESTROVET ${ }^{\circledR}$ ) was applied. On day 8, the DIB ${ }^{\circledR}$ device was removed plus $2 \mathrm{ml}$ of prostaglandin (SINCROCIO®) plus $0.5 \mathrm{ml}$ of estradiol cypionate (ESTROGAL ${ }^{\circledR}$ ) and $2 \mathrm{ml}(\mathrm{eCG})\left(\mathrm{NOVORMON}{ }^{\circledR}\right)$. The "T2" was similar to the previous protocol with the difference that no hormone (eCG) (NOVORMON®) was supplied. 50 hours after removal, the device was inseminated and $2 \mathrm{ml}$ of GnRh (CONCEPTASE) was applied. 40 days later, a pregnancy diagnosis was made with ultrasonography. An evaluation was made with an anova table. The results suggest that there is no statistically significant difference between the treated groups, but there are numerical differences in favor of treatment 1 . In conclusion, the use of the hormone equine chorionic gonadotropin increases the pregnancy rates.
\end{abstract}

Keywords: Estrous cycle, protocol, ovulation, heat

\footnotetext{
${ }^{\mathrm{i}}$ Bachiller en Ingeniería Zootecnista. Universidad Nacional Toribio Rodríguez de Mendoza de Amazonas. Correo electrónico: gucho_15@hotmail.com

${ }^{2}$ Docente de la Universidad Nacional Toribio Rodríguez de Mendoza de Amazonas. Correo electrónico: nilton.murga.epg@untrm.edu.pe
} 


\section{INTRODUCCIÓN}

Hoy en día la ganadería exige a los productores una mayor eficacia para dar mayor rentabilidad de las explotaciones de ganado vacuno, en esta realidad, la optimización de la eficiencia reproductiva es uno de los principales factores que contribuyen a mejorar las utilidades. No hay dudas que la tasa de preñez y sobre todo su distribución, tienen un impacto muy importante sobre la situación económica de un establecimiento de cría.

Baruselli et al., (2005) Conforme pasan los años se viene trabajando en el desarrollo de protocolos de inseminación artificial a tiempo fijo (IATF), lo que ha aumentado significativamente la cantidad de vacas incluidas en estos programas.

El uso de dispositivos de P4 en combinación con eCG ha sido utilizado en vacas en anestro postparto. La eCG tiene un efecto similar a la FSH y su administración al momento de retirar el implante puede estimular el crecimiento folicular y la producción de estrógenos que conduciría a una liberación preovulatoria de $\mathrm{LH}$ en un número importante de animales (Villa N, et al,. 2007). Buscando mejorar la detección de celo en los hatos ganaderos, se realizaron protocolos de sincroniza ción de ovulación, lo que, a su vez nos facilita la inseminación a un gran número de vacas en un lapso de tiempo determinado. Dichos tratamientos se denominan inseminación artificial a tiempo fijo (Bo et al., 2002).

A través de los años los protocolos para controlar el ciclo estral en la vaca evolucionaron favorablemente, probando diferentes hormonas y combinaciones de las mismas. Primero, se hicieron estudios sobre la utilización de progesterona exógena con el fin de prolongar la fase lútea. Años más tarde, decidieron utilizar una asociación de 2 estrógenos y gonadotropinas. En la actualidad, se han hecho estudios de las ondas foliculares que mostraron que la dinámica folicular se produce desde las primeras semanas de desarrollo embrionario (Motta P, 2011)

Se verificó que con la aplicación de eCG el día 8 de sincronización se consiguen cuerpos lúteos únicos de mayor tamaño, incrementando así la tasa de preñez (Nasser et al., 2004). De igual manera en un estudio realizado encontraron que con la aplicación de la eCG al día 8 se mejora la tasa de aprovechamiento ( Tovio N, 2011). En hembras receptoras cruzadas Bos Indicus X Bos Taurus. Se han realizado estudios utilizando eCG en donde se ha observado el incremento en las tasas de preñez en programas de inseminación artificial a tiempo fijo (IATF) (Baruselli et al., 2003; Bó, 2003; Sousa et al).

\section{MATERIAL Y MÉTODO}

El objeto de estudio fueron los protocolos de inseminación artificial con la utilización de semen fresco.

En la presente investigación se utilizó un diseño completamente al azar (DCA) con 2 tratamientos y 21 repeticiones, se realizó el análisis de varianza para determinar las diferencias significativas entre los tratamientos y la prueba de Duncan para comparar las medias de los tratamientos en los parámetros evaluados, se trabajó con un nivel de significancia de $95 \%$.

El universo de la población estuvo conformado por los 270 bovinos del fundo Santa Elena.

La muestra estuvo representada por 42 vacas, las cuales fueron seleccionadas previa evaluación transrectal de los ovarios.

El muestreo se realizó dividiendo en dos grupos: T1, 21 vacas con protocolo con eCG y T2, 21 vacas con protocolo sin eCG.

- Edad: 2.5 a 6 años.

- Número de partos: 1 a 4, no se tomará en cuenta las vaquillas.

- Ultimo parto: $\geq 90$ días.

- Corporal (CC): $\geq 2.5 \mathrm{y} \leq 4.0$.

Se evaluó previamente a 80 vacas del fundo Santa Elena a través de un examen transrectal para de esta manera evaluar si está en condiciones para ingresar al protocolo de inseminación a tiempo fijo de las cuales mediante un examen del sistema reproductivo mediante palpación transrectal para descartar animales con problemas y rechazar aquellos animales que no se encuentren dentro de los parámetros establecidos, dentro de las cuales 42 vacas fueron seleccionadas para ser sincronizadas y para posteriormente ser inseminadas.

\section{Tabla 1}

Protocolo de sincronización de celo con eCG.

\begin{tabular}{|c|c|}
\hline $\begin{array}{c}\text { Día } \\
\text { Proto } \\
\text { colo }\end{array}$ & Mañanas (8 a.m.) \\
\hline $\begin{array}{l}\text { Día } 0 \\
\text { Día } 1 \\
\text { Día } 2 \\
\text { Día } 3 \\
\text { Día } 4 \\
\text { Día } 5 \\
\text { Día } 6 \\
\text { Día } 7\end{array}$ & Colocar P4 (DIB0 $0.5 \mathrm{gr})+0.8 \mathrm{ml} \mathrm{BE}$ (Estrov)et \\
\hline Día 8 & $\begin{array}{l}\text { Retiro de P4 }+2.5 \mathrm{~m} 1 \mathrm{PG}(\text { Sincrocib eCG } \\
\text { (Novormoh) } 2 \mathrm{~m} 1+\mathrm{CE} \text { (Estroga) } 10.5 \mathrm{ml}\end{array}$ \\
\hline Día 10 & $\begin{array}{c}\text { IATF }+ \text { GnRh } \begin{array}{c}\text { (Conceptas } 2 \mathrm{ml}(50 \text { horas post retirc } \\
\text { delimplante DIB })\end{array}\end{array}$ \\
\hline
\end{tabular}

Fuente: Modificación del protocolo de Gabriel Bo (2002 


\section{$>$ Tratamiento 1}

El protocolo utilizado fue una modificación de Gabriel Bo y consistió en lo siguiente:

En el día 0 se insertó un dispositivo (DIB de 0.5 gr) junto con $0.8 \mathrm{ml}$ de Benzoato de Estradiol (Estrovet ${ }^{\mathrm{g}}$ ) por vía intramuscular ( para sincronizar el desarrollo folicular), se debe resaltar que el uso de estrógenos al comienzo del tratamiento fue con el fin de iniciar una atresia folicular ya existente y así impedir el desarrollo de folículos persistentes ya que baja considerablemente la fertilidad, una vez que los folículos se atresian emerge una nueva onda folicular a los 4 días, de esta manera se garantiza el surgimiento de un nuevo folículo y viable en el día que se retira el dispositivo intravaginal.

En el día 8 se retiró el dispositivo, se aplicó $2.5 \mathrm{ml}$ de prostaglandina (Sincrocio) esto para inducir luteólisis, se administró $0.5 \mathrm{ml}$ de cipionato de estradiol (Estrogal) para sincronizar ovulación, finalmente se administró $2 \mathrm{ml}$ de eCG (Novormon) que ayudó a una buena ovulación y a la formación de un cuerpo lúteo accesorio la cual incrementó las concentraciones de P4 en la sangre de la vaca así como el porcentaje de preñez.

Se realizó IATF a las 50 horas de retirado el implante junto con la administración de $2 \mathrm{ml} \mathrm{GnRh}$, para un aumento en los picos de LH y así tener una buena ovulación.

Se retiró el dispositivo el día 8 para de esta manera asegurar un mayor crecimiento del folículo dominante.

\section{Tabla2}

Protocolo de sincronización de celo sine $C G$.

\begin{tabular}{|c|c|}
\hline $\begin{array}{c}\text { Día } \\
\text { Protocolo }\end{array}$ & Mañanas (8 a.m.) \\
\hline Día 0 & Colocar P4 (DIB $0.5 \mathrm{gr}$ ) $+0.8 \mathrm{ml} \mathrm{BE}$ (Estrove) \\
\hline Día 1 & \\
\hline Día 2 & \\
\hline Día 3 & \\
\hline Día 4 & \\
\hline Día 5 & \\
\hline Día 6 & \\
\hline Día 7 & \\
\hline Día 8 & $\begin{array}{l}\text { Retiro de } \mathrm{P} 4+2.5 \mathrm{mlPG} \text { (Sincroßi) }+\mathrm{CE} \\
(\text { EstrogaP) } 0.5 \mathrm{ml}\end{array}$ \\
\hline Día 10 & $\begin{array}{c}\mathrm{ATF}+\mathrm{GnRh}(\text { Concepta\$d } 2 \mathrm{ml}(50 \text { horas post retiro } \\
\text { del implante DIB) }\end{array}$ \\
\hline
\end{tabular}

Fuente: modificación del protocolo de Gabriel Bo (2002).

\section{Tratamiento 2}

En el día 0 se insertó un dispositivo (DIB de 0.5 gr) junto con $0.8 \mathrm{ml}$ de Benzoato de Estradiol (Estrovet)por vía intramuscular ( para sincronizar el desarrollo folicular), es necesario enfatizar en el uso de estrógenos al comienzo del tratamiento para provocar la atresia de los folículos existentes e impedir de esta manera la formación de folículos persistentes que interfieren negativamente en la fertilidad, como la atresia es seguida de una nueva onda folicular a los 4 días, se asegura de esta manera la presencia de un folículo nuevo y viable en el momento de retirar el dispositivo.

En el día 8 se retiró el dispositivo, se aplicó $2.5 \mathrm{ml}$ de prostaglandina (Sincrocio) esto para inducir luteólisis, administrar $0.5 \mathrm{ml}$ de cipionato de estradiol (Estrogal) para sincronizar ovulación, en este protocolo no se aplicó la hormona gonadotrofina coriónica equina (eCG).

Se realizó IATF a las 50 horas de retirado el implante junto con la administración de $2 \mathrm{ml} \mathrm{GnRh}$, para un aumento en los picos de LH y así tener una buena ovulación.

La colecta de semen se realizó en un toro de raza Angus rojo el cual se encuentraba en la estación experimental de la Universidad Nacional Toribio Rodríguez de Mendoza-Pomacochas.

Para la colecta de semen se utilizó la técnica de la vagina artificial.

\section{RESULTADOS.}

\section{Figura 1}

Porcentaje de vacas preñadas y vacías utilizando el protocolocone $C G$.

\section{-VACAS PREÑADAS "VACAS VACIAS}

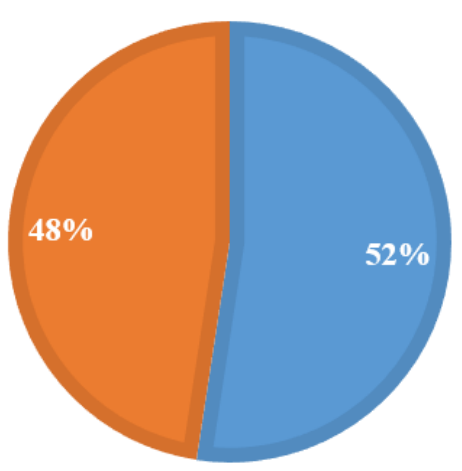

Se observa en la figura 1, la utilización de protocolo con hormona gonadotrofina coriónica equina el porcentaje de vacas preñadas es de $52 \%$ lo que es mayor que la cantidad de vacas vacías que es de $48 \%$. 


\section{Figura 2}

Porcentaje de vacas preñadas y vacías utilizando el protocolo sin $\mathrm{eCG}$.

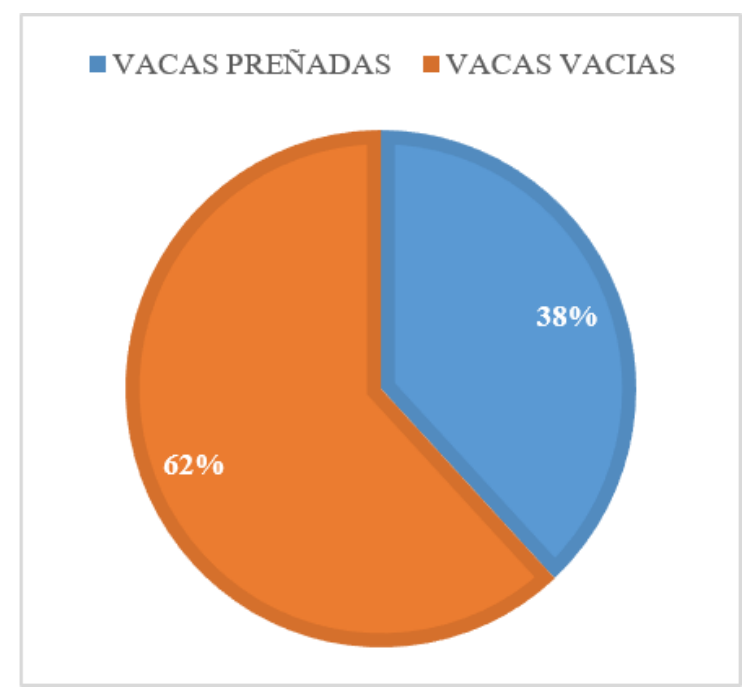

Se observa en el figura 2, que con la utilización de protocolo sin hormona gonadotrofina coriónica equina el porcentaje de vacas preñadas es de $38 \%$ lo que es menor que la cantidad de vacas vacías que es de $62 \%$.

\section{DISCUSIÓN}

Espinoza (2010), realizó un estudio, Para evaluar la respuesta de vacas sometidas a dos protocolos de sincronización de la ovulación. Al grupo uno (T1) se aplicó un implante intravaginal con 1,3 g de progesterona, más $2 \mathrm{mg}$ de benzoato de estradiol (BE); al retiro, siete días después, se administró 150 $\mathrm{mg}$ de D-Cloprostenol $(\mathrm{PgF} 2 \alpha)$ y, 24 horas más tarde, $1 \mathrm{mg}$ de benzoato de estradiol. Para el grupo 2 (T2), se realizó un protocolo similar al primero, con la diferencia que al día 7 se aplicó 500 UI de gonadotrofina coriónica equina (eCG) y al momento de la inseminación, se aplicó $100 \mathrm{mg}$ de gonadorelina $(\mathrm{GnRH})$. Todas fueron inseminadas 56 horas después de retirado el implante y el diagnóstico de preñez se dio a los 50 días de la inseminación artificial a tiempo fijo (IATF). Se encontró diferencias estadísticas significativas entre los dos tratamientos $(\mathrm{p}<0,05)$ en favor del T2 (eCG $+\mathrm{GnRH})$ con una tasa de preñez del $69 \%$ con respecto al T1 que fue del $36 \%$. En la investigación realizada, utilizando el mismo protocolo, en donde se administró la eCG al día 8, y con el diagnóstico de preñez a los 40 días post inseminación, se obtuvo el $52.4 \%$ de tasa de preñez, menor a la tasa de preñez presentada por Espinosa.

En el trabajo de investigación que se desarrolló se utilizaron 2 protocolos modificado de Gabriel Bo, en donde en el primer protocolo se llevaron a cabo las siguientes actividades: día cero se colocó el DIV (DIB) y se les aplicó $0,8 \mathrm{ml}$ de Benzoato de estradiol (Estrovet $^{\mathrm{a}}$ ) y en el día $8 \mathrm{se}$ les retiro el dispositivo y se inyectaron $2.5 \mathrm{ml}$ de prostaglandina (Sincrocio), junto con $0.5 \mathrm{ml}$ de Cipionato de estradiol (Estrogal) y $2 \mathrm{ml}$ eCG (NOVORMON). Con respecto al segundo protocolo es similar al primero, con la diferencia que no se aplica la hormona gonadotrofina coriónica equina (eCG) (NOVORMON). Se obtuvo una tasa de concepción de $52.4 \%$ con respecto al protocolo endone se utilizó eCG. Así mismo (Fernández et al., 2017) utilizando un protocolo en donde se aplicó eCG, obtuvo una tasa de concepción de $45 \%$, menor al resultado obtenido en éste proyecto de tesis.

El presente trabajo de investigación tuvo como objetivo determinar la tasa de concepción a través del uso de eCG en un lote de 42 animales bos indicus en el fundo Santa Elena, Bagua grande en donde dichas vacas fueron servidas mediante la técnica biotecnológica del uso de semen fresco, se obtuvo un resultado con una tasa de concepción de $45.2 \%$ estos resultados fueron comprobados a través del uso de la ultrasonografía evaluados a los 40 días después de la inseminación. Estos resultados fueron superados por (Ezequiel, 2015). Quien realizó un trabajo de IATF con semen fresco vs semen crioprecervado en donde se utilizó: semen fresco (790 vientres) y semen crioprecervado (519 vientres), los resultados obtenidos del análisis, mostraron diferencia significativa, obteniéndose un porcentaje de preñez de $66 \%$ y $55.23 \%$ respectivamente, se concluye que el uso de semen fresco en los rodeos evaluados, logro incrementar los porcentajes de preñez obtenidos en IATF, de manera significativa sobre el uso de semen crioprecervado.

\section{CONCLUSIONES}

Los porcentajes de preñez obtenidos para el T1 IATF con eCG fue de $52.4 \%$ y para el T2 IATF sin eCG fue del $38.1 \%$, lo que nos lleva a la conclusión de que el protocolo en el que se utilizó eCG presentó una mayor tasa de concepción.

No se determinó diferencias estadísticas significativas entre los tratamientos, pero se observó que el tratamiento con hormona gonadotrofina coriónica equina (eCG) presentó mayor porcentaje de vacas preñadas.

La eficacia reproductiva óptima es crucial para incrementar los rendimientos netos. Sin embargo, la variabilidad de las respuestas a los tratamientos tradicionales y el tiempo y esfuerzo que se requieren para realizar la detección del celo han afectado al rendimiento reproductivo de los rodeos. La incorporación de técnicas diseñadas para controlar la dinámica de la onda folicular y la ovulación en los últimos años ha reducido los problemas asociados con la detección del celo. Además, los tratamientos 
con dispositivos de liberación de progesterona, estradiol y eCG han brindado la posibilidad de aplicar la IATF con altas tasas de preñez en vacas cíclicas y no cíclicas.

\section{REFERENCIAS BIBLIOGRÁFICAS.}

Bó et al. (2002). Tratamientos hormonales para inseminación artificial a tiempo fijo en bovinos para carne: algunas experiencias realizadas en Argentina. Sitio Argentino de Producción Animal, 1-14. Recuperado el 15 de Octubre de 2019, de http://www.produccion-animal.com .ar/informacion_tecnica/inseminacion_artifici al/26-tratamientos_hormonales_ia_tiempo fijo.pdf

Baruselli P, B. G. (2005). Introduccion de la IATF en el manejo reproductivo de rebaños de ganado de engorde en brasil. intervet, 4-30.

Ezequiel L,.(2015) Comparación entre uso de semen fresco versus congelado en programas de IATF. Universidad nacional de Córdoba Faculta de Ciencias Agropecuarias, Córdoba.

Fernandez F,. (2017). Porcentaje de Concepción en Vacas Bos indicus Utilizando Sincronización de Estro e Inseminación Artificial a Tiempo fijo (IATF). REDVET, 1-3. Obtenido de https:// www.google.com/url?sa=t\&source $=$ web\&rct $=\mathrm{j} \&$ url=http://www.iracbiogen.com.ar/admi $\mathrm{n} /$ biblioteca/documentos/t.f.outlopepe.docx\& ved=2ahUKEwiojuK

Tivio N, (2011)Efectos de la aplicación de eCG (Día 5 u 8) sobre el desarrollo del cuerpo lúteo, nivel de progesterona y tasa de preñez en hembras receptoras de embriones bovinos. . Universidad Nacional de Colombia, Bogotá., D.C. Colombia.

Espinosa M,. (2010). Efectos de diferentes protocolos para IATF sobre la tasa de preñez aplicados en ganado lechero. Facultad de ciencias agropecuarias escuela para graduados Universidad Nacional de Córdoba, Córdoba, iran. Obtenido de https://www .google.com/url?sa=t\&source=web\&rct=j\&ur $\mathrm{l}=\mathrm{http}: / /$ www.iracbiogen.com.ar/admin/biblio
teca/documentos/Trabajo\%2520Final\%2520 Marcia $\% 252$

Motta P,. (2011). dinámica folicular en la vida reproductiva de la hembra bovina. vet.zootec., 98- 99. Villa N, et al C. A. (2007). Evaluación de Cuatro Protocolos de Sincronización Para Inseminación a Tiempo Fijo en Vacas Bos indicus Lactantes. Rev. Cient. (Maracaibo) v.17 n.5 Maracaibo oct. 2007, 11-12 